\title{
Effect of ticagrelor on the serum level of hs-CRP, ESM-1 and short-term prognosis of patients with acute STEMI
}

\author{
PENG WEI ${ }^{1,2}$, BING HAN $^{1}$, WEN-JUN ZHANG ${ }^{3}$, JIE BAI $^{3}$, CHUN-YING JIANG $^{1}$, CHONG-RONG QIU $^{4}$, \\ QIAN ZHANG ${ }^{1}$, QIANG FU ${ }^{1}$ and XIANG-JUN YANG ${ }^{2}$
}

\author{
${ }^{1}$ Department of Cardiology, Xuzhou Central Hospital, The Affiliated Hospital of Southeast University, Xuzhou, \\ Jiangsu 221009; ${ }^{2}$ Department of Cardiology, The First Affiliated Hospital, Soochow University, Suzhou, Jiangsu 215006; \\ ${ }^{3}$ Department of Geriatrics, Changhai Hospital of The Second Military Medical University, Shanghai 200433; \\ ${ }^{4}$ Department of Cardiology, People's Hospital of Ganzhou, Ganzhou, Jiangxi 341000, P.R. China
}

Received August 22, 2016; Accepted November 14, 2016

DOI: $10.3892 /$ etm.2016.3987

\begin{abstract}
The aim of the present study was to observe and investigate the changes in the serum level of high-sensitivity $\mathrm{C}$-reactive protein (hs-CRP), the endothelial cell-specific molecule-1 (ESM-1) and short-term prognosis of patients with ST-segment elevation myocardial infarction (STEMI) treated by ticagrelor. We enrolled 107 patients with acute STEMI who were admitted in the Department of Cardiology for the first time with occurrence of symptoms, and we successfully performed emergency operation of percutaneous coronary intervention. The patients were divided into two groups, 54 patients in the ticagrelor group (treatment group) and 53 patients in the clopidogrel group (control group), according to the administration of ticagrelor or clopidogrel in dual anti-platelet therapy. Then, we observed the changes at the time of admission, at $24 \mathrm{~h}$, and 4th and 7th day after administration and investigated the correlations between them and the effect of ticagrelor on the short-term prognosis of acute STEMI patients. Significant increases of the serum levels of hs-CRP and ESM-1 were seen in patients of the two groups $24 \mathrm{~h}$ after administration of drugs with statistically significant differences between the groups $(\mathrm{P}<0.05)$, and on the 4 th and 7 th day we found a downward trend with statistically significant differences $(\mathrm{P}<0.05)$. The level of ESM-1 enhanced the increase of hs-CRP, indicating there was
\end{abstract}

Correspondence to: Dr Qiang Fu, Department of Cardiology, Xuzhou Central Hospital, The Affiliated Hospital of Southeast University, 199 Jiefang Road, Xuzhou, Jiangsu 221009, P.R. China E-mail: pennsylwestern@163.com

Dr Xiang-Jun Yang, Department of Cardiology, The First Affiliated Hospital, Soochow University, 188 Shizi Street, Suzhou, Jiangsu 215006, P.R. China

E-mail: yang_xiangjun@163.com

Key words: ticagrelor, acute ST-segment elevation myocardial infarction, percutaneous coronary intervention, high-sensitivity C-reactive protein, endothelial cell-specific molecule-1 a positive correlation between ESM- 1 and hs-CRP ( $\mathrm{r}=0.535$, $\mathrm{P}<0.001)$. A comparison of the occurrence rates of ischemic outcome event, bleeding and overall adverse events between the two groups yielded no statistically significant difference $(\mathrm{P}>0.05)$. In conclusion, the present study demonstrates that ticagrelor can reduce the prevalence of inflammatory reactions rapidly and stabilize the functions of vascular endothelium to improve the stability of atherosclerosis plaque and decrease the occurrence rate of thrombosis as well as ischemic outcome event without any obvious increase in the risk of bleeding. Thus, ticagrelor should be recommended in clinical practices for the treatment of patients with STEMI.

\section{Introduction}

As the most severe type of acute coronary syndrome (ACS), acute ST-segment elevation of myocardial infarction (STEMI), with the characteristics of acute onset, poor prognosis and high mortality (1), is caused by the vascular endothelial injury and rupture of inflammatory and unstable plaque resulted by coronary atherosclerosis, which can progress to thrombosis, leading to acute occlusion of culprit vessels to severe myocardial ischemia, even to myocardial necrosis. At present, myocardial ischemia-reperfusion, through reopening the myocardial infarction related arteries by percutaneous coronary intervention (PCI), has become one of the major procedures in the treatment of STEMI (2). Particular attention has been shifted to the research on the pathogenesis of STEMI, how to prevent thrombosis, reduce the inflammatory reaction, stabilize the characteristics of plaques and improve the vascular endothelial functions to preserve the survived myocardium.

In this study, the objective was set to observe the serum level changes of high-sensitivity C-reactive protein (hs-CRP), a major inflammatory marker reflecting the stability of plaque, and the endothelial cell-specific molecule-1 (ESM-1), the newly found biological marker reflecting the degree of disturbance of endothelial functions of patients with acute STEMI. Our results confirm the correlation between hs-CRP and ESM-1 and the effect of ticagrelor on the short-term prognosis of acute STEMI patients, which serves as reference for research 
in effective therapy to alleviate the inflammatory reaction, stabilize the endothelial functions and reduce the prevalence of adverse events of cardiovascular system.

\section{Materials and methods}

Clinical material. We continuously enrolled 107 patients who were admitted to the Xuzhou Center Hospital (Jiangsu, China) between December 2013 and January 2015 for acute STEMI, accepted the procedure of PCI and met the inclusion criteria. There were 62 males and 45 females aged 30-70 years. After obtaining written informed consent, we randomly divided these patients into two groups according to the administration of ticagrelor or clopidogrel during the therapy, 54 patients in the ticagrelor group (treatment group: Load dosage of $180 \mathrm{mg}$, maintenance dosage of $90 \mathrm{mg} /$ time, twice a day) and 53 patients in the clopidogrel group (control group: Load dosage of $300 \mathrm{mg}$, maintenance dosage of $75 \mathrm{mg} / \mathrm{time}$, once a day), and performed follow-up by telephone for 30 days after the discharge of the enrolled patients. All the cases corresponded with the definition of acute myocardial infarction of the Third Global Seminar of ESC/ACCF/AHA/WHF and the Diagnostic Criteria of Acute Myocardial Infarction stipulated by the Chinese Society of Cardiology $(2010)(3,4)$. Exclusion criteria were: i) Patients who would have severe adverse reaction to ticagrelor or were resistant to clopidogrel; ii) patients with a history of myocardial infarction or with other heart diseases and/or severe heart failure (NYHA III or IV); iii) patients who had severe hepatic or renal dysfunction; iv) patients who had history of PCI or coronary artery bypass grafting (CABG) or had accepted other types of surgeries or been injured recently; v) patients with endocrine diseases such as diabetes, and immune system diseases such as acute or chronic infections, digestive tract infections, hematological diseases, malignant tumors, rheumatic disease of connective tissues; vi) patients with severe hypertension without any drug controls, cerebrovascular diseases or peripheral vascular disease; and vii) patients who had any conditions deemed inappropriate by researchers for inclusion in the study.

Baseline material. The baseline material of patients included the gender, age, body mass index (BMI), risk factors of cardiology [smoking, intake of alcohol, hypertension, family medical history of coronary heart disease, the level of total cholesterol, low-density lipoprotein (LDL), high-density lipoprotein (HDL) and triglyceride], the drugs utilized during the study (administration of low-molecular-weight heparin, IIb/IIIa glycoprotein inhibitors, $\beta$-receptor inhibitor, ACEI, statins, CCB, nitrates, PPIs, H2RA), count of platelet, levels of glutamic oxaloacetic transaminase (GOT), glutamic-pyruvic transaminase (GPT), creatinine, urea nitrogen, blood glucose under stress and ejection fraction (EF).

Measurement of $h s-C R P$. Venous blood $(5 \mathrm{ml})$ was drawn from the elbow of each subject at the time of admission, $24 \mathrm{~h}$ after administration of drugs and $5 \mathrm{ml}$ of fasting venous blood was drawn from the same site of patients in the morning of the 4th and 7th day after administration. The supernatant of samples was collected for analysis by BN II Automatic Analyzer of Protein (Siemens AG, Munich, Germany) and measurement of hs-CRP by chemiluminescence with the measurement agent of hs-CRP.

Measurement of ESM-1. Venous blood (5 ml) was drawn from the elbow of each subject at the time of admission, $24 \mathrm{~h}$ after administration of drugs and $5 \mathrm{ml}$ of fasting venous blood was drawn from the same site of patients in the morning of the 4th and 7th day after administration. Samples were required to be in a supine position for $\geq 20 \mathrm{~min}$ without any procedures for anti-coagulation and then immediately placed at $4^{\circ} \mathrm{C}$ to coagulate for $1 \mathrm{~h}$. Samples were centrifuged at $1,760 \mathrm{x}$ for $15 \mathrm{~min}$ under $4^{\circ} \mathrm{C}$, and the serum were drawn into EP tubes and stored at $-80^{\circ} \mathrm{C}$ for measurement. The expression level of EMS-1 was measured by ELISA, and the ELISA kits were provided by Shanghai Enzyme-linked Biotechnology Co., Ltd. (Shanghai, China).

Observation of primary efficacy endpoint. A 30-day follow-up was performed for the patients in the two groups and the items for statistical analysis included the following: i) The occurrence rate of ischemic endpoint events, including cardiac death, acute myocardial infarction, symptoms with the urgent need of performing coronary revascularization, or stroke; and ii) occurrence rate of bleeding events, i.e., identifying the occurrence rate of major safety endpoint events (bleeding) during hospitalization and in 30 days after surgery according to PLATO, including major bleeding events (life-threatening or lethal events), other major bleeding events, secondary bleeding events and slight bleeding events, i.e., other bleeding events that did not need any medical intervention, such as bruise, gingival bleeding, bleeding at the injection site (5).

Statistical analysis. Data analysis was performed using SPSS 22.0 (SPSS, Inc., Chicago, IL, USA). Quantitative data were presented as mean \pm standard deviation (SD). A normality test was conducted for quantitative data. If the data were correspondent with the normal distribution, an independent $\mathrm{t}$-test was applied in difference analysis of quantitative data of two groups; if not correspondent with normal distribution, the Mann-Whitney rank sum test was utilized for the difference analysis of quantitative data of two groups. Quantitative data were presented as cases or percentage $(n / \%)$, tested by $\chi^{2}$ test or Fisher's exact test. Difference with $\mathrm{P}<0.05$ was considered statistically significant. For correlation analysis among variables, if the distribution of variables were correspondent with bivariate normal distribution, Pearson's product-moment correlation analysis was performed and if not correspondent with bivariate normal distribution or ranked data, Spearman's rank correlation analysis was applied. $\mathrm{P}<0.05$ was considered to indicate a statistically significant difference.

\section{Results}

Comparison of baseline material in the groups. In the two groups, no statistically significant differences $(\mathrm{P}>0.05)$ were found following a comparison of gender, age, BMI, risk factors of cardiology (smoking, intake of alcohol, hypertension, family medical history of coronary heart disease, the level of total cholesterol, LDL, HDL and triglyceride). The results obtained from different treatments during the study (administration of 
Table I. Comparison of baseline material of patients in the two groups.

\begin{tabular}{|c|c|c|c|}
\hline Variables & $\begin{array}{l}\text { Treatment } \\
\text { group } \\
(\mathrm{N}=54)\end{array}$ & $\begin{array}{l}\text { Control } \\
\text { group } \\
(\mathrm{N}=53)\end{array}$ & P-value \\
\hline Gender (male/female) & $31 / 23$ & $31 / 22$ & 0.910 \\
\hline Age (years) & $53.28 \pm 11.85$ & $52.49 \pm 11.69$ & 0.730 \\
\hline BMI $\left(\mathrm{kg} / \mathrm{m}^{2}\right)$ & $24.80 \pm 2.55$ & $24.21 \pm 2.46$ & 0.226 \\
\hline \multicolumn{4}{|l|}{$\begin{array}{l}\text { Risk factors of } \\
\text { cardiology, n (\%) }\end{array}$} \\
\hline Smoking & $26(48.1)$ & $25(47.2)$ & 0.919 \\
\hline Intake of alcohol & $30(55.6)$ & 27 (50.9) & 0.633 \\
\hline Hypertension & $21(38.9)$ & $24(45.3)$ & 0.503 \\
\hline $\begin{array}{l}\text { Family medical history } \\
\text { of coronary heart disease }\end{array}$ & $5(9.3)$ & $4(7.5)$ & 1.000 \\
\hline $\mathrm{TC}(\mathrm{mmol} / \mathrm{l})$ & $4.88 \pm 0.55$ & $4.75 \pm 0.66$ & 0.290 \\
\hline LDL-C (mmol/l) & $3.09 \pm 0.41$ & $3.18 \pm 0.40$ & 0.272 \\
\hline $\mathrm{TG}(\mathrm{mmol} / \mathrm{l})$ & $1.35 \pm 0.43$ & $1.33 \pm 0.43$ & 0.785 \\
\hline \multicolumn{4}{|l|}{$\begin{array}{l}\text { Drugs utilized during } \\
\text { the study, } \mathrm{n}(\%)\end{array}$} \\
\hline $\begin{array}{l}\text { Low molecular } \\
\text { weight heparin }\end{array}$ & $54(100.0)$ & $53(100.0)$ & - \\
\hline $\begin{array}{l}\text { IIb/IIIIa glycoprotein } \\
\text { inhibitors }\end{array}$ & $24(44.4)$ & $23(43.4)$ & 0.913 \\
\hline$\beta$-receptor inhibitor & $43(79.6)$ & $45(84.9)$ & 0.475 \\
\hline ACEI/ARB & $41(75.9)$ & $40(75.4)$ & 1.000 \\
\hline Statins & $54(100.0)$ & $53(100.0)$ & 1.000 \\
\hline $\mathrm{CCB}$ & $18(33.3)$ & $19(35.8)$ & 0.784 \\
\hline Nitrates & $47(87.0)$ & $45(84.9)$ & 0.751 \\
\hline PPIs & $28(51.9)$ & $30(56.6)$ & 0.622 \\
\hline H2RA & $13(24.1)$ & $12(22.6)$ & 0.861 \\
\hline $\operatorname{GOT}(\mu / 1)$ & $39.39 \pm 8.50$ & $41.45 \pm 6.96$ & 0.174 \\
\hline GPT $(\mu / 1)$ & $34.56 \pm 5.45$ & $35.50 \pm 6.76$ & 0.433 \\
\hline Creatinine $(\mu \mathrm{mol} / \mathrm{l})$ & $88.38 \pm 11.09$ & $85.11 \pm 9.90$ & 0.111 \\
\hline Urea nitrogen $(\mathrm{mmol} / \mathrm{l})$ & $6.88 \pm 1.65$ & $6.55 \pm 1.92$ & 0.343 \\
\hline $\begin{array}{l}\text { Blood glucose under } \\
\text { stress }(\mathrm{mmol} / \mathrm{l})\end{array}$ & $7.67 \pm 2.30$ & $7.99 \pm 2.42$ & 0.483 \\
\hline $\mathrm{EF}$ & $0.50 \pm 0.04$ & $0.49 \pm 0.06$ & 0.511 \\
\hline
\end{tabular}

No statistically significant differences were found in comparison to baseline material in the groups $(\mathrm{P}>0.05)$. BMI, body mass index; LDL, low-density lipoprotein; GOT, glutamic oxaloacetic transaminase; GPT, glutamic-pyruvic transaminase; EF, ejection fraction.

low-molecular-weight heparin, IIb/IIIa glycoprotein inhibitors, $\beta$-receptor inhibitor, ACEI, statins, CCB, nitrates, PPIs, H2RA), count of platelet, levels of GOT, GPT, creatinine, urea nitrogen, blood glucose under stress and EF are presented in Table I.

Comparison of serum levels of hs-CRP and ESM-1 in the two groups. No statistically significant differences were found in the comparison of serum levels of hs-CRP and ESM- 1 between the two groups at the time of admission, but significant

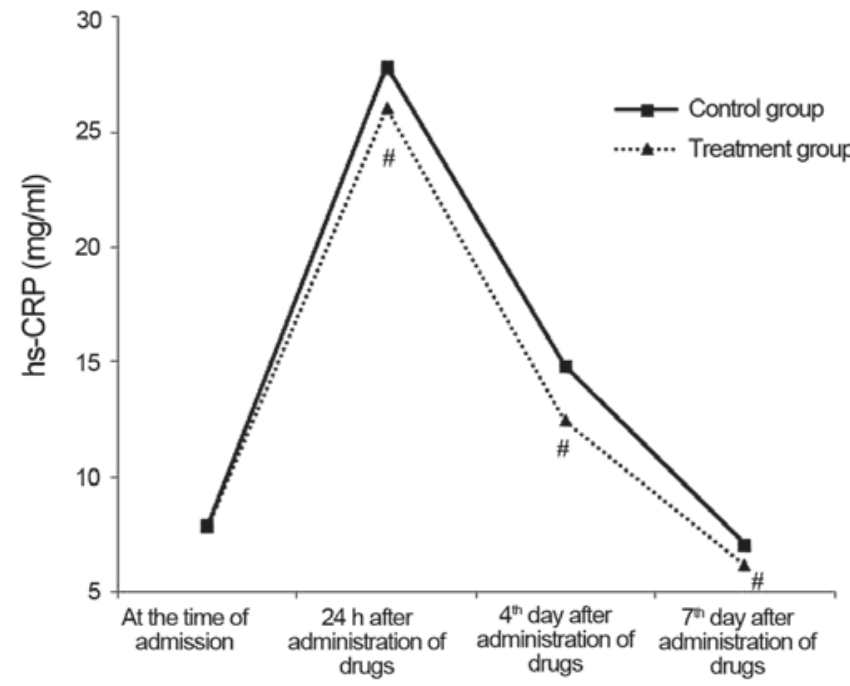

Figure 1. Comparison of serum level of hs-CRP of patients at different time-points in the groups. ${ }^{\#}$ Comparison between treatment and control groups $(\mathrm{P}<0.05)$. hs-CRP, high-sensitivity $\mathrm{C}$-reactive protein.

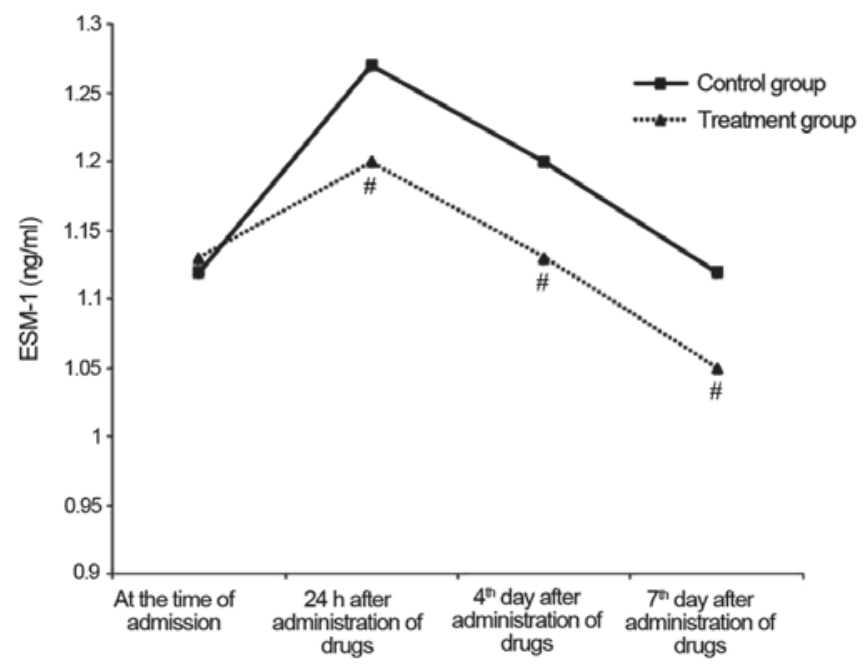

Figure 2. Comparison of serum level of ESM-1 of patients at different time-points in the groups. ${ }^{\#}$ Comparison between treatment and control groups $(\mathrm{P}<0.05)$. ESM-1, endothelial cell-specific molecule-1.

increases of serum levels of hs-CRP and ESM-1 were seen in patients of the two groups $24 \mathrm{~h}$ after administration of drugs with statistically significant differences between the groups $(\mathrm{P}<0.05)$, and in the 4th and 7th day we found a downward trend with statistically significant differences $(\mathrm{P}<0.05)$ (Table II and Figs. 1 and 2).

Correlation between serum levels of ESM-1 and hs-CRP of patients at the time of admission. To investigate the correlation between serum levels of ESM-1 and hs-CRP, we performed Spearman's bivariate analysis. The level of ESM-1 was enhanced with the increase of inflammatory factors, indicating there was a positive correlation between ESM-1 and hs-CRP ( $\mathrm{r}=0.535$, P <0.001) (Table III and Fig. 3).

Comparison of major efficacy endpoint events of 30 days in the two groups. In comparison of the occurrence rates of 
Table II. Comparison of serum levels of hs-CRP and ESM-1 in the groups.

\begin{tabular}{lrr}
\hline Variables & Treatment group $(\mathrm{N}=54)$ & Control group $(\mathrm{N}=53)$ \\
\hline hs-CRP (mg/l) & & $7.91 \pm 2.72$ \\
At the time of admission & $7.83 \pm 2.84$ & $27.89 \pm 6.50$ \\
24 h after administration of drugs & $26.07 \pm 5.34$ & $14.81 \pm 5.51$ \\
4th day after administration of drugs & $12.46 \pm 4.00$ & $7.03 \pm 2.31$ \\
7th day after administration of drugs & $6.15 \pm 1.67$ & 0.002 \\
ESM-1 (mg/l) & & $1.12 \pm 0.18$ \\
At the time of admission & $1.13 \pm 0.15$ & $1.27 \pm 0.19$ \\
24 h after administration of drugs & $1.20 \pm 0.18$ & $1.20 \pm 0.18$ \\
4th day after administration of drugs & $1.13 \pm 0.17$ & $1.12 \pm 0.16$ \\
7th day after administration of drugs & $1.05 \pm 0.16$ & 0.806 \\
\hline
\end{tabular}

hs-CRP, high-sensitivity C-reactive protein; ESM-1, endothelial cell-specific molecule-1.

Table III. Analysis of the correlation among hs-CRP and ESM-1.

\begin{tabular}{lr}
\hline Variables & hs-CRP \\
\hline ESM-1 & \\
r & 0.535 \\
P-value & $<0.001$ \\
\hline
\end{tabular}

hs-CRP, high-sensitivity C-reactive protein; ESM-1, endothelial cell-specific molecule-1.

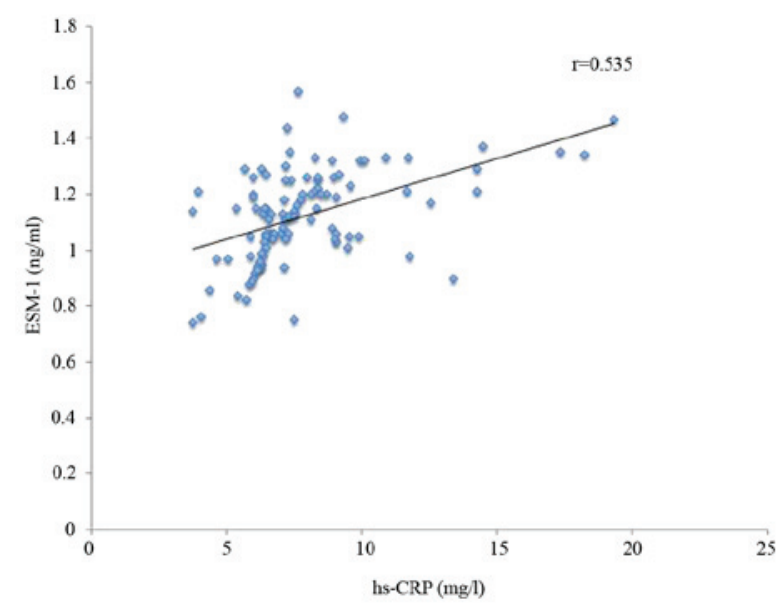

Figure 3. Scatter diagram of correlation between serum levels of hs-CRP and ESM-1. hs-CRP, high-sensitivity C-reactive protein; ESM-1, endothelial cell-specific molecule-1.

ischemic outcome events, bleeding and overall adverse events between the groups, we found no statistically significant difference $(\mathrm{P}>0.05)$ (Table IV).

\section{Discussion}

Ticagrelor, one of the newly developed cyclo-pentyl-triazolopyrimidines (CPTPs), is an oral preparation of $\mathrm{P} 2 \mathrm{Y} 12$ receptor
Table IV. Comparison of major efficacy endpoint events of 30 days in the two groups.

\begin{tabular}{lccc}
\hline & $\begin{array}{c}\text { Treatment } \\
\text { group } \\
\text { Variables }\end{array}$ & $\begin{array}{c}\text { Control } \\
\text { group } \\
(\mathrm{N}=54)\end{array}$ & P-value \\
\hline $\begin{array}{l}\text { Ischemic endpoint } \\
\text { events, n (\%) }\end{array}$ & $1(1.9)$ & $3(5.7)$ & 0.363 \\
Cardiac death & $1(1.9)$ & $1(1.9)$ & 1.000 \\
Acute myocardial & $0(0.0)$ & $1(1.9)$ & 1.000 \\
infarction & & & \\
Emergency coronary & $0(0.0)$ & $0(0.0)$ & - \\
revascularization & & & \\
$\quad$ Stroke & $0(0.0)$ & $1(1.9)$ & 1.000 \\
Bleeding events, n $(\%)$ & $5(9.3)$ & $4(7.5)$ & 1.000 \\
Major bleeding events & $0(0.0)$ & $0(0.0)$ & - \\
Other major bleeding events & $0(0.0)$ & $0(0.0)$ & - \\
$\begin{array}{l}\text { Secondary bleeding events } \\
\text { Slight bleeding events }\end{array}$ & $1(1.9)$ & $1(1.9)$ & 1.000 \\
Total adverse events, n $(\%)$ & $4(7.4)$ & $3(5.7)$ & 1.000 \\
\hline
\end{tabular}

antagonist. Compared with other P2Y12 receptor antagonists such as clopidogrel or prasugrel, ticagrelor could, without metabolic activation, exert its effect immediately after oral administration with a median peak-reaching time of $1.5 \mathrm{~h}$, and combine with the P2Y12 receptor reversibly, resulting in a rapid recovery of platelets after drug withdrawal. Additionally, they affected the migration of inflammatory cells to exert other pharmacological effects through suppression on P2Y12 receptor distributed on the surface of inflammatory cells such as microglial cells, neutrophils, dendritic cells and mononuclear macrophages $(6,7)$.

As an indicator of inflammation with high sensitivity and specificity, hs-CRP interacted with vascular endothelium cells and other cells to accelerate the process of vascular inflammatory reaction, making the plaques inside the coronary artery more unstable, even rupture, leading to a series of pathological 
and physiological processes such as leukocyte adhesion, activation and oxidation of platelets and newly developed thrombosis. hs-CRP has served as an independent predictive factor for ACS and is of significance in identification of inflammatory reaction, predicating the stability of plaque and cardiac events $(8,9)$. In this study, we found that serum level of hs-CRP of treatment group was lower than that of control group ay $24 \mathrm{~h}$, and 4th and 7th day after administration of drugs with statistically significant differences $(P<0.05)$, indicating the superiority of ticagrelor to the clopidogrel in suppressing the vascular inflammatory reactions. Anti-platelet drugs effectively restrained the process of atheromatous plaques not only by inhibiting the coagulation of platelet and thrombosis, but also possibly through the improvement in ischemic and oxygen-deficient environment and suppression on the immune inflammatory reactions.

First found and reported in 1996, ESM-1, also known as Endocan, is mainly secreted by endothelial cells (10). Research has shown that ESM-1 may be a new biological marker for endocrine function disorder. Lassalle et al found that ESM-1 was highly expressed in atheromatous plaques, speculating that the increase in secretion of ESM-1 may promote the migration and proliferation of vascular smooth muscle cells (VSMCs) and that ESM-1 may play a role in the pathology of atherosclerosis (11). Kose et al (12) reported that an increase of ESM-1 expression was seen in the subjects, inferring that ESM-1 may be a new biological marker for endothelial pathology. Furthermore, Tadzic et al confirmed that the decrease of ESM-1 could reduce the activation of endothelial cells, thus delaying the progress of atherosclerosis (13). In the present study, we found that ticagrelor and clopidogrel alleviated the effect of ESM-1 in varying degrees, and statistically significant differences were found in comparison of ESM-1 in ticagrelor group and clopidogrel group at $24 \mathrm{~h}$, and 4th and 7 th day after administration of drugs $(\mathrm{P}<0.05)$, indicating that ticagrelor could better improve the endothelial functions.

To confirm the role of ESM-1 in the progress of STEMI, we performed a correlation analysis for ESM-1 and hs-CRP, and it was found that the level of ESM-1 enhanced the increase of inflammatory factors, indicating there was a positive correlation between ESM- 1 and hs-CRP $(r=0.535$, $\mathrm{P}<0.001)$. Thus, ESM-1, the marker of endothelial function disorder, and inflammatory factors such as hs-CRP participated in the cause and progression of coronary heart disease and acute events of coronary artery, in which ESM-1 activated the emission of inflammatory factors such as hs-CRP, and inflammatory factors in turn, promoted the expression of ESM-1. This has rarely been reported in the literature. Thus, future investigations are to examine the correlation between ESM- 1 and ACS.

In this study, we found no statistically significant differences $(\mathrm{P}>0.05)$ in comparison of occurrence rate of ischemic endpoint events in 30 days in two groups, which was consistent with the results in literature (14), possibly due to the small number of samples. In addition, there was no statistically significant difference in comparison of occurrence rate of bleeding events $(\mathrm{P}>0.05)$, suggesting that no significant increase was seen in risk of bleeding events in ticagrelor group compared to the clopidogrel group and ticagrelor is safe and effective for patients.
In conclusion, ticagrelor, in the treatment of patients with STEMI, can decrease the level of inflammatory factors, reduce the prevalence of inflammatory reactions rapidly and stabilize the functions of vascular endothelium to improve the stability of atherosclerosis plaque and decrease the occurrence rate of thrombosis as well as ischemic outcome events without any obvious increase in the risk of bleeding. Thus, ticagrelor should be recommended in clinical practice.

\section{References}

1. Armstrong PW, Gershlick AH, Goldstein P, Wilcox R, Danays T, Lambert Y, Sulimov V, Rosell Ortiz F, Ostojic M, Welsh RC, et al; STREAM Investigative Team: Fibrinolysis or primary PCI in ST-segment elevation myocardial infarction. N Engl J Med 368: 1379-1387, 2013

2. Pedersen F, Butrymovich V, Kelbæk H, Wachtell K, Helqvist S, Kastrup J, Holmvang L, Clemmensen P, Engstrøm T, Grande P, et al: Short- and long-term cause of death in patients treated with primary PCI for STEMI. J Am Coll Cardiol 64: 2101-2108, 2014.

3. Thygesen K, Alpert JS, Jaffe AS, Simoons ML, Chaitman BR, White HD, Thygesen K, Alpert JS, White HD, Jaffe AS, et al; Writing Group on the Joint ESC/ACCF/AHA/WHF Task Force for the Universal Definition of Myocardial Infarction; ESC Committee for Practice Guidelines (CPG): third universal definition of myocardial infarction. Eur Heart J 33: 2551-2567, 2012.

4. Tubaro M, Danchin N, Goldstein P, Filippatos G, Hasin Y, Heras M, Jansky P, Norekval TM, Swahn E, Thygesen K, et al: Pre-hospital treatment of STEMI patients. A scientific statement of the Working Group Acute Cardiac Care of the European Society of Cardiology. Acute Card Care 13: 56-67, 2011.

5. James SK, Roe MT, Cannon CP, Cornel JH, Horrow J, Husted S, Katus H, Morais J, Steg PG, Storey RF, et al; PLATO Study Group: Ticagrelor versus clopidogrel in patients with acute coronary syndromes intended for non-invasive management: substudy from prospective randomised PLATelet inhibition and patient Outcomes (PLATO) trial. BMJ 342: d3527, 2011.

6. Diehl P, Olivier C, Halscheid C, Helbing T, Bode C and Moser M: Clopidogrel affects leukocyte dependent platelet aggregation by P2 $\mathrm{Y}_{12}$ expressing leukocytes. Basic Res Cardiol 105: 379-387, 2010.

7. Webster CM, Hokari M, McManus A, Tang XN, Ma H, Kacimi R and Yenari MA: Microglial P2Y12 deficiency/inhibition protects against brain ischemia. PLoS One 8: e70927, 2013.

8. Taniguchi H, Momiyama Y, Ohmori R, Yonemura A, Yamashita T, Tamai S, Nakamura H and Ohsuzu F: Associations of plasma C-reactive protein levels with the presence and extent of coronary stenosis in patients with stable coronary artery disease. Atherosclerosis 178: 173-177, 2005.

9. Kirbis S, Breskvar UD, Sabovic M, Zupan I and Sinkovic A: Inflammation markers in patients with coronary artery disease - comparison of intracoronary and systemic levels. Wien Klin Wochenschr 122 (Suppl 2): 31-34, 2010.

10. Béchard D, Gentina T, Delehedde M, Scherpereel A, Lyon M, Aumercier M, Vazeux R, Richet C, Degand P, Jude B, et al: Endocan is a novel chondroitin sulfate/dermatan sulfate proteoglycan that promotes hepatocyte growth factor/scatter factor mitogenic activity. J Biol Chem 276: 48341-48349, 2001.

11. Lassalle P, Molet S, Janin A, Heyden JV, Tavernier J, Fiers W, Devos R and Tonnel AB: ESM-1 is a novel human endothelial cell-specific molecule expressed in lung and regulated by cytokines. J Biol Chem 271: 20458-20464, 1996.

12. Kose M, Emet S, Akpinar TS, Kocaaga M, Cakmak R, Akarsu M, Yuruyen G, Arman Y and Tukek T: Serum endocan level and the severity of coronary artery disease: a pilot study. Angiology 66: 727-731, 2015.

13. Tadzic R, Mihalj M, Vcev A,Ennen J, Tadzic A and Drenjancevic I: The effects of arterial blood pressure reduction on endocan and soluble endothelial cell adhesion molecules (CAMs) and CAMs ligands expression in hypertensive patients on Ca-channel blocker therapy. Kidney Blood Press Res 37: 103-115, 2013.

14. Wallentin L, Becker RC, Budaj A, Cannon CP, Emanuelsson H, Held C, Horrow J, Husted S, James S, Katus H, et al; PLATO Investigators: Ticagrelor versus clopidogrel in patients with acute coronary syndromes. N Engl J Med 361: 1045-1057, 2009. 